\title{
Le sfide alla salute psicofisica delle famiglie italiane nel periodo dell'emergenza Covid-19: uno studio pilota sull'impatto del burnout genitoriale in diverse categorie professionali
}

\author{
Teresa Di Fiore* ${ }^{*}$ Teresa Galanti*, Gloria Guidetti* , Daniela Marchetti* Paolo Roma**, \\ Maria Cristina Verrocchio* , Michela Cortini* ${ }^{*}$ Stefania Fantinelli* \\ * Dipartimento di Scienze Psicologiche, della Salute e delTerritorio, Università “G. d'Annunzio", Chieti-Pescara; \\ ** Dipartimento di Neuroscienze Umane, Università La Sapienza, Roma
}

\begin{abstract}
Obiettivi: valutare l'impatto dell'epidemia da Covid-19 sulla genitorialità in famiglie con bambini in età pediatrica ed esaminare come il burnout genitoriale possa essere influenzato dalla presenza di uno o più figli con diagnosi certificata di patologia conclamata fisica o psicologico-psichiatrica.

Metodi: campione composto da 970 genitori con figli in età pediatrica che hanno risposto a un questionario sul burnout genitoriale.

Risultati: le madri hanno un livello di burnout più elevato. Emerge un effetto principale significativo rispetto al lavoro dei genitori: i lavoratori manuali presentano livelli di burnout più alti durante il lockdown. La presenza di figli con diagnosi certificata di patologia risulta essere un predittore del burnout.

Conclusioni: il pediatra di base dovrebbe attenzionare i casi a rischio che minano la salute psicofisica dei bambini, vigilando sul deterioramento delle cure genitoriali sia nelle famiglie con casi di patologia sia in quelle in condizioni di salute buone.
\end{abstract}

\begin{abstract}
Objectives: to assess the impact of the Covid-19 pandemicon parenting in families with pediatric age childrenand examine how parental burnout can be influenced by the presence of one or more children with certified diagnosis of full-blown physical or psychological-psychiatric pathology.
\end{abstract}

Methods: the sample consisted of 970 parents with pediatric age children who were administered a questionnaire on parental burnout.

Results: mothers have a higher level of burnout. A significant main effect emerged with respect to the parents' work: manual workers have higher burnout levels during quarantine. The presence of children with a certified diagnosis is a predictor of parental burnout.

Conclusions: the primary pediatrician should care of potentially risky situations that undermine children mental and physical health, especially by supervising the decline of parental care both in families with cases of manifest pathology and in those in good health conditions.

\section{Introduzione}

In letteratura sono già presenti diversi studi che resocontano come e quanto la popolazione pediatrica possa più o meno essere soggetta a contrarre il Covid-19. Da tali studi risulta che, in linea con quanto già rilevato in Cina, l'incidenza della malattia si attesta intorno allo $0,5 \%$ nei bambini fino a 9 anni e all'1\% nel resto della popolazione pediatrica $[1,2]$.

Sono in modo particolare $\mathrm{i}$ dati riferiti al tasso di mortalità e alla severità della manifestazione della malattia (che, di fatto, sembra risparmiare la popolazione pediatrica e adolescenziale) che hanno spinto tanti pediatri e ricercatori a spostare il proprio sguardo dagli aspetti più strettamen- te clinici legati al rischio Covid-19 a effetti sanitari cosiddetti collaterali, riconducibili all'emergenza sanitaria Covid-19.

Potremmo, in tal senso, identificare almeno 3 linee di rischio e relativo intervento. In primis, va sicuramente citato il sovvertimento degli approcci clinico-assistenziali consolidati, sia per i servizi sanitari pubblici sia per i medici delle cure primarie, provocato dalla rapidità del diffondersi della pandemia. Questo aspetto, per quanto ovvio, ha indubbiamente delle ripercussioni a livello sanitario; si pensi, solo per fare un esempio, all'aumento della consulenza medico-pediatrica via telefono, con tutte le sfide comunicativo-relazionali che questa comporta, soprattutto riguardo alla diffi- coltà nel formulare una diagnosi e nel garantirsi l'engagement dei genitori dei piccoli pazienti in termini di adesione alle cure. In secondo luogo, in tutto il periodo del lockdown, a fronte di un tasso minore di richiesta di intervento sanitario-pediatrico (abbastanza ovvio dovuto anche alla minore esposizione a rischi), ha destato preoccupazione il ritardo con cui tante famiglie sono ricorse a interventi sanitari per problemi di salute varia (non necessariamente collegati al Covid-19) per paura di contrarre il virus in ospedale. Diverse unità pediatriche hanno registrato l'arrivo di pazienti con notevole ritardo rispetto all'insorgenza di sintomi (e questo non solo per quanto riguarda sintomi Covid-19 bensì, più in generale, per sintomi di diverse patologie, anche gravi e potenzialmente letali). Questo dato sposta la preoccupazione del pediatra di base, quando intercettato, verso la sfida di far capire ai genitori che possono essere tante le malattie più rischiose e potenzialmente letali rispetto alla paura di contrarre il $\mathrm{Co}^{-}$ vid-19 negli ospedali [3].

Deve essere poi considerato l'aspetto ascrivibile all'impatto psicologico che la paura del virus e il nuovo stile di vita cui ci ha costretti può provocare sulle famiglie e sui bambini e adolescenti. I primi studi evidenziano un livello elevato di distress psicologico nella popolazione italiana [4]. Circa un quarto delle famiglie italiane ha almeno un figlio minore in casa. Diventa quindi essenziale chiedersi come le reazioni psicologiche degli adulti di convivere con la minaccia del virus possa incidere negativamente sui figli minori.

Ultimo aspetto molto importante è anche il passaggio allo smartworking imposto a molti genitori a causa delle restrizioni messe in atto dal Governo per arginare i possibili contagi e la diffusione della pandemia. Questo cambiamento repentino, detta- 
to dalle necessità del momento, ha alterato gli equilibri personali, lavorativi e familiari, rappresentando un potenziale agente stressogeno in grado di minacciare il benessere psicologico. Un'indagine interessante condotta su Linkedin che ha coinvolto 2.000 partecipanti mostra chiaramente gli effetti del lavoro da casa dettati dal lockdown e l'impatto che ha sul benessere psicologico. Quasi la metà del campione ha riferito di sentirsi più ansioso o stressato per la nuova modalità di lavoro e che in media ha lavorato più ore [5]. Questo dato evidenzia come, nella situazione attuale, sia minato il confine tra mondo del lavoro e vita familiare, e il più ampio concetto di work-life balance, ovvero il mantenimento di un equilibrio tra vita professionale e personale/familiare. A questo, poi, va aggiunto il timore, espresso da una parte considerevole del campione, di perdere il proprio posto di lavoro o di essere licenziati. Questa percezione di job insecurity, che di per sé rappresenta un potente agente stressogeno, si aggiunge e aggrava un quadro già molto sfidante dal punto di vista psicologico [6].

Anche una recente ricerca condotta negli Stati Uniti ha già ipotizzato che le condizioni create dalla pandemia possano aumentare i fattori di rischio di burnout genitoriale [7]; in particolare ci si riferisce alla mancanza di lavoro, all'insicurezza economica, alla mancanza di supporto sociale e alla mancanza di tempo libero.

A livello internazionale vi è una maggioranza di studi interessati al burnout lavorativo durante il periodo di lockdown, con un focus particolare sulle professioni sanitarie; pertanto il nostro studio offre un significativo punto di vista nuovo.

\section{Obiettivi}

Questo studio ha lo scopo di fornire uno sguardo d'insieme sulla salute della famiglia. La nostra ricerca parte proprio da qui; da quanto è stato considerato da altri l'altra facciata della medaglia Covid-19, quella appunto ascrivibile alla salute e al benessere psicosociale [2]. Secondo studi recenti [8], i pazienti in età pediatrica costretti a periodi di quarantena hanno una probabilità di sviluppare stress post traumatico 4 volte superiore a soggetti di altra età. In quanto psicologi, il primo spunto di riflessione che vogliamo fornire riguarda un aspetto che a noi sembra fondamentale, ossia osservare la salute del paziente pediatrico inserendola in un più generale quadro di salute della famiglia. Crediamo, infatti, che solo un approccio olistico, capace di osservare congiuntamente bambini e genitori possa essere vincente in termini sia preventivi che di intervento.

L'obiettivo del presente studio è quello di testare l'incidenza dello stress genitoriale nella popolazione adulta al tempo dell'emergenza Covid-19, cercando di leggerne le implicazioni pediatriche con un taglio critico. In altre parole, partendo da un focus sull'incidenza del burnout genitoriale, ci interessa sollevare domande e spunti per la pratica pediatrica, facendo riflettere il lettore sul ruolo psicosociale cui il pediatra è chiamato in questa emergenza sanitaria. Abbiamo analizzato il tasso di stress genitoriale al termine del primo mese di lockdown, suddividendo il campione in funzione della presenza o assenza di uno o più figli con una diagnosi certificata di patologia conclamata (sia di tipo fisico che di tipo psicologico-psichiatrico). L'ipotesi dalla quale partiamo è che i genitori di bambini in età pediatrica affetti da patologia manifestano un tasso superiore di burnout genitoriale. I fattori causali che maggiormente impattano sullo sviluppo del burnout sono legati ad alcuni cambiamenti sociali che hanno modificato il concetto di genitore nel corso del tempo: in particolare una perdita di rigidità e autorità. A questo si aggiungono due aspetti: il primo riguarda la maggiore attenzione posta sui diritti dei bambini e sui doveri dei genitori che devono fronteggiare più richieste di attenzione da parte dei figli; il secondo, la maggiore diffusione del lavoro femminile [9].

E stato recentemente dimostrato che, come un'esposizione duratura allo stress può condurre al burnout lavorativo, così un'esposizione duratura a un eccessivo stress percepito nell'espletamento delle funzioni genitoriali può condurre al burnout genitoriale. Il burnout genitoriale è una sindrome unica e contesto-specifica che si sviluppa a seguito di una lunga ed eccessiva esposizione allo stress genitoriale cronico [10] e quindi quando vi è uno squilibrio cronico tra richieste e risorse [11]. Per richieste, in questo specifico contesto, si intendono tutti i fattori che in qualche modo possono favorire l'aumento dello stress, come: gli innumerevoli compiti intrinseci al ruolo di genitore, la mancanza di supporto esterno, ma anche caratteristiche individuali del genitore e il ricorso a pratiche educative incoerenti e rigide $[12,13]$. Il burnout genitoriale è un costrutto multifattoriale che comprende tre dimensioni. La dimensione principale del parental burnout è l'esaurimento emotivo caratterizzato dalla sensazione di svuotamento e sopraffazione del proprio ruolo genitoriale [14]. I genitori emotivamente esauriti avvertono estrema fatica nell'esercizio del proprio ruolo, non dispongono di sufficiente energia per occuparsi dei loro figli e sentono che occuparsi dei figli ha esaurito ogni loro risorsa; ciò implica sentirsi emotivamente scarichi e stanchi al risveglio mattutino, avere la sensazione di non farcela più, sentire che essere genitore richiede troppo impegno e percepire i propri figli come più “difficili” [15]. La seconda dimensione è la distanza emotiva dal proprio/dai propri figlio/figli che implica il sentirsi sempre meno coinvolti nell'educazione/accadimento dei figli, fare il minimo indispensabile per loro e limitare le interazioni agli aspetti funzionali a discapito degli aspetti emotivi. La terza dimensione è la perdita del senso di efficacia nel proprio ruolo di genitore che è definita dal sentirsi come se non si fosse in grado di gestire $i$ problemi con calma e in maniera efficiente e dal non provare più piacere nello stare con i propri figli.

Dal punto di vista psicologico ci sono alcuni tratti di personalità che possono predisporre al burnout: elevati livelli di nevroticismo sono un fattore di rischio [13] e infatti il burnout è spesso accompagnato da stress ed emozioni negative. Ulteriore fattore di rischio è rappresentato dalle condizioni ambientali, per esempio il numero di figli e l'assenza di supporto sociale.

La letteratura specializzata si sta recentemente interessando agli effetti negativi derivanti dal burnout genitoriale. Alcune ricerche hanno evidenziato associazioni con sintomi depressivi, disturbi del sonno, dipendenze, conflitto di coppia, ideazione suicidaria $[11,12,16,17]$. Inoltre, sebbene le conseguenze del burnout genitoriale sui figli non sono state ancora studiate direttamente, è stata sottolineata un'associazione significativa tra l'esaurimento emotivo genitoriale e l'aumento dei comportamenti trascuranti e violenti nei confronti dei figli $[16,18]$.

\section{Metodi}

Il campione che abbiamo raggiunto è un campione di convenienza, contattato attraverso i principali social media (Facebook, WhatsApp, ecc.), costituito da adulti con almeno un figlio in età pediatrica. Abbiamo contattato 1.220 persone; di queste, hanno risposto al nostro questionario, gestito da piattaforma Qualtrics per garantire l'anonimato, 970 soggetti, tutti di nazionalità italiana, residenti in diverse aree del nostro Paese. I principali dati socio-familiari sono riportati nella Tabella 1.

Il campione è costituito prevalentemente $\mathrm{da}$ soggetti di genere femminile (844 vs 126), con un'età media di 39,6 anni (D.S. 6,5), con un range di età compresa tra 23 e 67 anni. Su base volontaria, nelle prime due settimane di aprile, hanno compilato un questionario sul burnout genitoriale.

Il burnout genitoriale è stato misurato con il Parental Burnout Inventory (PBI), un questionario self-report di 22 item su scala 


\begin{tabular}{|c|c|c|c|c|c|c|c|c|c|}
\hline \multicolumn{10}{|c|}{ Titolo di studio } \\
\hline \multicolumn{2}{|c|}{ Licenza elementare } & \multicolumn{2}{|c|}{$\begin{array}{l}\text { Diploma di scuola media } \\
\text { inferiore }\end{array}$} & \multicolumn{2}{|c|}{$\begin{array}{c}\text { Diploma di scuola media } \\
\text { superiore }\end{array}$} & \multicolumn{2}{|c|}{ Laurea } & \multicolumn{2}{|c|}{ Titolo post laurea } \\
\hline \multicolumn{2}{|c|}{4} & \multicolumn{2}{|c|}{39} & \multicolumn{2}{|c|}{343} & \multicolumn{2}{|c|}{343} & \multicolumn{2}{|c|}{241} \\
\hline \multicolumn{10}{|c|}{ Stato civile } \\
\hline \multicolumn{2}{|c|}{ Celibe/nubile } & \multicolumn{2}{|c|}{ Coniugato/a } & \multicolumn{2}{|c|}{ Separato/a o divorziato/a } & \multicolumn{2}{|c|}{ Vedovo/a } & \multicolumn{2}{|c|}{ Convivente } \\
\hline \multicolumn{2}{|c|}{32} & \multicolumn{2}{|c|}{709} & \multicolumn{2}{|c|}{73} & \multicolumn{2}{|c|}{4} & \multicolumn{2}{|c|}{152} \\
\hline \multicolumn{10}{|c|}{ Chi vive in casa con lei? (possibilità di scegliere più opzioni) } \\
\hline $\begin{array}{l}\text { Il padre/ } \\
\text { la madre } \\
\text { di mio/a } \\
\text { figlio/a }\end{array}$ & $\begin{array}{l}\text { II/la mio/a } \\
\text { compagno/a }\end{array}$ & $\begin{array}{l}\text { La nonna } \\
\text { paterna }\end{array}$ & $\begin{array}{l}\text { Il nonno } \\
\text { paterno }\end{array}$ & $\begin{array}{l}\text { La nonna } \\
\text { materna }\end{array}$ & $\begin{array}{l}\text { Il nonno } \\
\text { materno }\end{array}$ & $\begin{array}{l}1 \text { o più } \\
\text { bisnonni }\end{array}$ & $\begin{array}{l}\text { Tata/go- } \\
\text { vernante/ } \\
\text { babysitter }\end{array}$ & Nessuno & Altri parenti \\
\hline 815 & 85 & 12 & 9 & 36 & 26 & 4 & 4 & 71 & 23 \\
\hline \multicolumn{10}{|c|}{ Come definirebbe la condizione socio-economica del suo nucleo familiare? } \\
\hline \multicolumn{2}{|c|}{ Bassa $(0-15.000)$} & \multicolumn{3}{|c|}{ Medio bassa $(16.000-33.000)$} & \multicolumn{3}{|c|}{ Medio alta (34.000-55.000) } & \multicolumn{2}{|c|}{ Alta (oltre 55.000) } \\
\hline \multicolumn{2}{|c|}{99} & \multicolumn{3}{|c|}{423} & \multicolumn{3}{|c|}{349} & \multicolumn{2}{|c|}{99} \\
\hline \multicolumn{10}{|c|}{ Con chi sta trascorrendo la misura di contenimento del contagio? (possibilità di scegliere più opzioni) } \\
\hline Da solo & Con partner & Con i figli & \multicolumn{3}{|c|}{$\begin{array}{l}\text { Con uno o più membri della famiglia } \\
\text { di origine }\end{array}$} & Con co & uilini non del & amiglia & Altro \\
\hline 0 & 802 & 970 & & 91 & & & 1 & & 10 \\
\hline & & & È st & o contagia & /a dal Cov & $19 ?$ & & & \\
\hline & & Sì & & & & & No & & \\
\hline & & 7 & & & & & 963 & & \\
\hline & $\mathrm{Ha}$ & ufruito (o & usufruer & o) di un sc & egno psic & gico o di & a psicoter & ia? & \\
\hline & Sì, in passato & & & Sì, att & mente & & & No & \\
\hline & 211 & & & & & & & 701 & \\
\hline
\end{tabular}

Likert a sette punti che valuta le dimensioni di esaurimento emotivo, distanza emotiva e perdita di capacità ed efficacia genitoriale con punteggi più alti che indicano livelli più elevati di burnout. Esempi di item sono: "Mi sento emotivamente prosciugato dal mio ruolo genitoriale", "A volte mi sento come se mi stessi prendendo cura dei miei figli con il pilota automatico", "Non riesco più a dimostrare amore ai miei figli" [19]. Il PBI ha dimostrato buone proprietà psicometriche in studi su popolazioni di genitori $[19,20]$ e ha consentito di definire la specificità del burnout genitoriale rispetto al burnout lavoro-correlato, ma anche rispetto alla depressione o al più generale stress sperimentato dai genitori nell'accudimento. Tuttavia, esistono ancore poche evidenze sull'utilizzo di un cut-off che consenta di individuare la presenza di burnout genitoriale. Nel presente studio il punteggio totale del PBI ha mostrato un livello di attendibilità adeguato e buoni indici di normalità, con asimmetria e curtosi più che soddisfacenti. Per tale ragione, abbiamo proseguito applicando la statistica parametrica dell'Anova fattoriale (Box 1) per testare l'effetto del genere e dell'avere o meno in famiglia un minore con diagnosi di malattia fisica o psichiatrica. Abbiamo, inoltre, effettuato un'Anova fattoriale con cui abbiamo testato i diversi livelli di burnout nei genitori, distinti per genere e per tipo di lavoro. Come tipologia di lavoro, abbiamo distinto i disoccupati, dividendo poi i lavoratori in manuali e cognitivi.

\section{Risultati e discussione}

Le madri presentano un livello significativamente superiore rispetto ai padri di burnout genitoriale $(\mathrm{F}=4,48 ; \mathrm{p}<.03)$. Questo risultato non stupisce, visto che nella nostra cultura i compiti di cura dei figli risultano ancora piuttosto sbilanciati investendo maggiormente le madri.

La presenza di un bambino con diagnosi è un predittore di burnout genitoriale in una fase di emergenza sanitaria quale quella che stiamo vivendo $(F=5,86 ; \mathrm{p}<.01)$. Nonostante non ci sia un effetto di interazione statisticamente significativo, colpisce che siano le madri a manifestare meno burnout dei padri nei casi di patologia manifesta nel figlio (Figura 1).

Risulta interessante, in termini di effetti principali, anche il ruolo svolto dal lavoro.

\section{BoX 1. Anova}

L'Analisi della Varianza è una tecnica statistica di analisi dei dati che permette di verificare ipotesi relative a differenze tra medie di due 0 più campioni tenendo conto contemporaneamente di più variabili [21]. Si definiscono variabili indipendenti quelle variate in modo sistematico dallo sperimentatore e variabili dipendenti quelle che vengono osservate e misurate.

Infatti, nonostante non ci sia un effetto statisticamente significativo che distingua tra lavoratori e non lavoratori e tra diversi tipi di lavoratori, emerge un trend in cui i lavoratori manuali sembrano presentare un maggiore livello di burnout nel periodo del lockdown. Questo dato può probabilmente essere compreso facendo riferimento al fatto che i lavoratori manuali sono stati quelli maggiormente deprivati del lavoro, che, sappiamo, può avere un ruolo importante in termini di equilibrio mentale per quanto concerne il doppio ruolo di genitore e lavoratore. Chi era disoccupato prima del Covid-19 continua probabilmente a esserlo anche oggi e chi lavorava cognitivamente prima del Covid-19 continua a lavorare, 


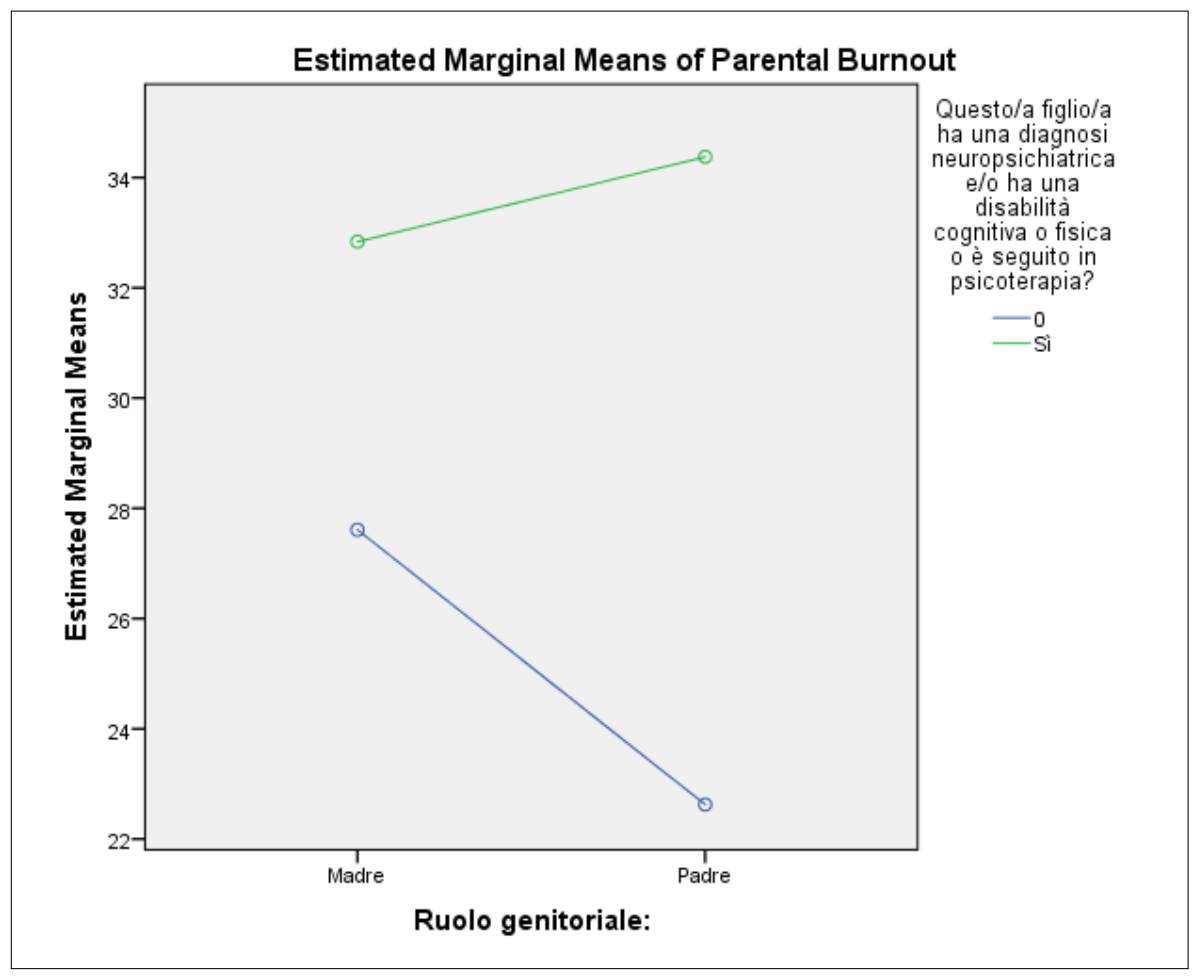

Figura 1. Livelli di burnout genitoriale in presenza di patologia manifesta del figlio.

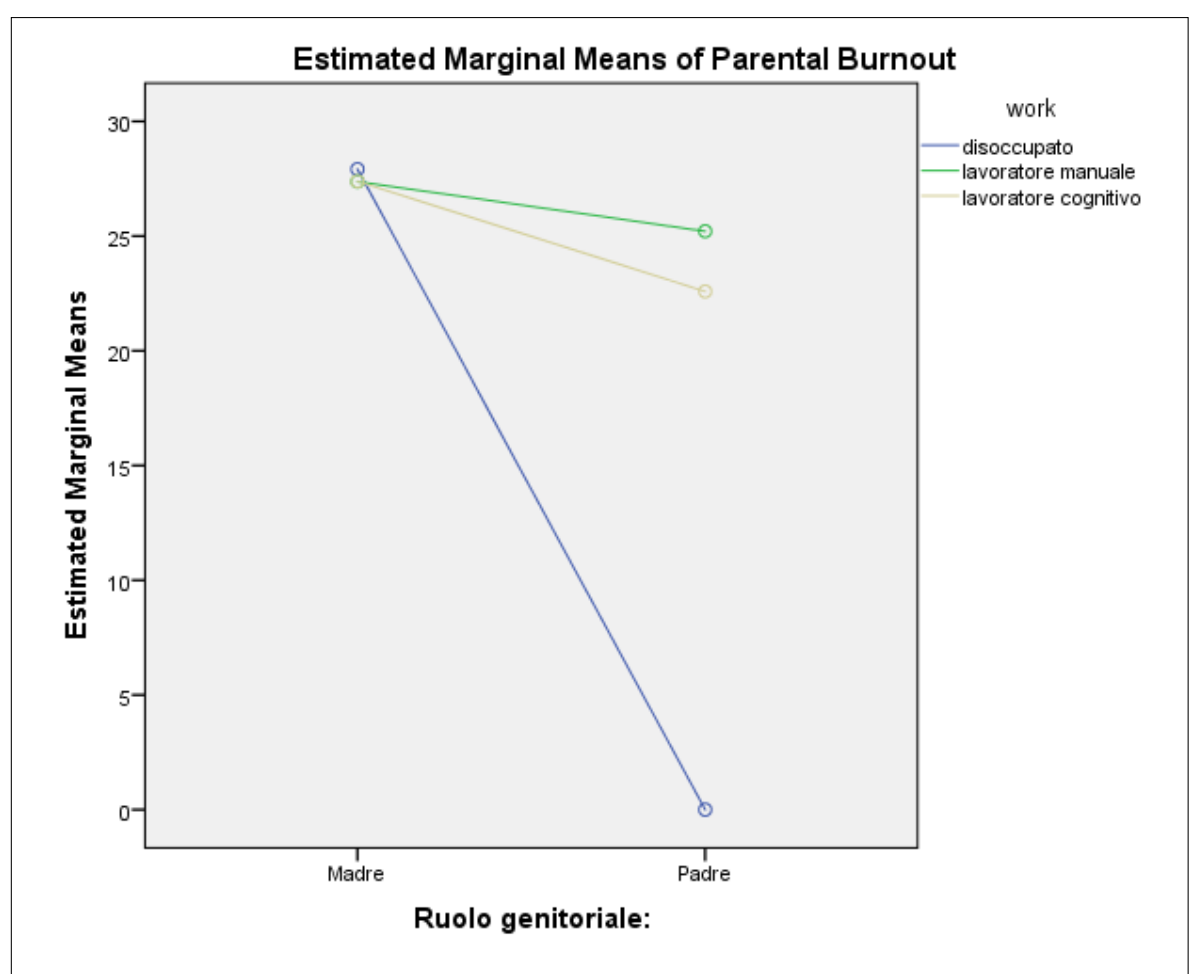

Figura 2. Livelli di burnout genitoriale e condizione lavorativa dei genitori.

sebbene a distanza e con nuove modalità (che peraltro potrebbero essere indagate, rappresentando il telelavoro, alle volte, più un fattore di rischio che un fattore protettivo). I lavoratori manuali, invece, per esempio gli artigiani o gli operai, hanno smesso di lavorare da un giorno all'altro; qualcuno potrà aver beneficiato della cassa integrazione, qualcun altro probabilmente no; in ogni caso, tutti i lavoratori manuali, abituati anche a una certa fatica fisica, di colpo si sono ritrovati a non far nulla, con l'aggiun- saurimento di un genitore comporta necessariamente un aumentato carico nell'altro genitore. Ulteriore rischio è dato dal fatto che i bambini sono in stretta sintonia con le emozioni e gli stati d'animo dei genitori, e quindi un genitore a rischio di burnout potrebbe influenzare negativamente l'umore e la salute psicologica dei propri figli che potranno sentirsi minacciati dalla situazione nuova. Una famiglia che vive una condizione di burnout non è capace di fornire al bambino le cure e la vicinanza emotiva necessarie, con tutto ciò che questo implica a livello di salute pediatrica.

\section{Conclusioni}

Perché il nostro studio interroga il pediatra di base? Proviamo a vederlo nel dettaglio.

Quando si parla di stress post-traumatico o di malessere psicologico nel bambino, i primi attori a essere chiamati in causa sono i genitori, ai quali la società si rivolge come primi paladini protettori della salute dei propri figli. Quando però il genitore si ammala, a sua volta, di stress, può manifestare il burnout genitoriale che, come abbiamo spiegato, si palesa con un detrimento nella capacità di cura del bambino e con un pericoloso distanziamento emotivo dal bambino. Diventano dunque fondamentali, in questi casi, i ruoli degli insegnanti, degli psicologi e di tutte le altre figure genitoriali "aggiuntive" che ruotano attorno alla vita di un bambino.

Tra queste ultime, anche il pediatra di base, può e deve svolgere un ruolo delicato e importante, ossia fungere da "sentinella delle singole situazioni con maggiore difficoltà e disagio, a partire dai bambini fragili, più vulnerabili, per diverse cause" [2]. Questo dimostra, peraltro, come la professione del pediatra non possa essere ridotta alla sola dimensione sanitaria, coinvolgendo aspetti socio-psicologici di estrema importanza. Il nostro studio interroga il pediatra di base, chiamato a vigilare, nel dettaglio, in quelle situazioni di rischio che abbiamo evidenziato, con attenzione particolare alle famiglie che presentano casi di patologia manifesta ma anche a quelle famiglie con bambini in età pediatrica in buona condizione di salute che potrebbero risentire di un impoverimento delle cure genitoriali.

Come tutti gli studi empirici cross-sectional, anche il nostro risente dell'impossibilità di indagare in maniera longitudinale la dimensione del parental burnout; pertanto non è possibile effettuare un confronto con dati relativi a valutazioni pre-post lockdown.

$\bowtie$ teresa.difiore@unich.it

La bibliografia è consultabile online. 


\section{Bibliografia}

1.Tagarro A, Epalza C, Santos M, et al. Screening and severity of coronavirus disease 2019 (Covid-

19) in children in Madrid, Spain. JAMA Pediatr 2020,Apr 8 [Epubahead of print].

2. Guiducci C, Marchetti F. Covid-19 e bambini: il punto dalla letteratura al 18 marzo. Medico e Bambino. 2020;39(3):151-3.

3. Lazzerini M, Barbi E, Apicella A, Marchetti F, CardinaleF, \&amp;Trobia G. Delayed access or provision of care in Italyresulting from fear of COVID-19. The Lancet Child \&amp; Adolescent Health. 2020; 4(5), e10-e11.

4. Mazza C, Ricci E, Biondi S, Colasanti M, Ferracuti S, Napoli C, \&amp; Roma P. A Nationwide survey of psychological distress among Italian people during the COVID-19 pandemic: Immediate psychological responses and associated factors. International Journal of Environmental Research and Public Health. 2020; 17,3165.

5.Le conseguenze del lavoro da casa sugliitaliani in lockdown (2020), https://www.agi.it/cronaca/news/2020-05-14/smart-working-conseguenze-lavoratori-8611222/ .Visitato il 16 Maggio 2020.

6. Castellini, G, Lozza, E, Castiglioni, C, Chirumbolo, A. La misura della job insecurity: Revisione della scala di Chirumbolo et al. (2015). Rassegna di Psicologia. 2016; 4, $29-38$.

7. Griffith AK. Parental Burnout and Child MaltreatmentDuring the COVID-19 Pandemic (2020). Journal of Family Violence. Pubblicato online il 23 giugno 2020.

8. Sprang G, Silman M.Posttraumatic stress disorder in parents and youth after health-related disasters. DisasterMed Public. 2013;7: 105-110.

9. Della Rocca Federico, La Sindrome del "Burnout genitoriale": quando essere genitori porta all'esaurimento.https://www.usi.it/notizie/276/la-sindrome-del-burnout-genitoriale-quando-esseregenitori-porta-allesaurimento

10. Mikolajczak, M., Gross, J. J. \&amp;Roskam, I. (2019). Parental Burnout: What Is It and What Does It Matter? ClinicalPsychological Science 2019, Vol. 7(6) 1319- 1329

11. Mikolajczak, M., \&amp;Roskam, I. (2018). A theoretical and clinical framework for parental burnout: the balance between risks and resources (BR2). Frontiers in Psychology, 9, 886. https://doi.org/10.3389/ fpsyg.2018.00886.

12. Kawamoto, T., Furutani, K., \&amp;Alimardani, M. (2018). Preliminary validation of Japanese version of the parental burnout inventory and its relationship with perfectionism. Frontiers of Psychology, 9, 970.

13. Le Vigouroux, S., Scola, C., Raes, M.E., Mikolajczak, M. \&amp;Roskam, I. (2017). The big five personality traits and parental burnout: protective and risk factors. Personality and Individual Differences, 119 (2017) 216-219

14. Roskam, I., Raes, M.E. \&amp;Mikolajczak, M. (2017). Exhausted parents: Development and preliminary validation of the Parental burnout inventory. Frontiers in psychology, 8:163.doi: 10.3389/fpsyg.2017.00163

15. Gillis, A. \&amp;Roskam, I. (2019). Regulation between daily exhaustion and support in parenting: A dyadic perspective. International Journal of Behavioural Development, 1-10. DOI:

$10.1177 / 01650254198685367$

16. Mikolajczak, M., Brianda, M. E., Avalosse, H., \&amp;Roskam, I. (2018a). Consequences of parental burnout: A preliminary investigation of escape and suicidal ideations, sleep disorders, addictions, marital conflicts, child abuse and neglect. Child Abuse and Neglect, 80, 134-145.

https://doi.org/10.1016/j.chiabu.2018.03.025.

17. Mikolajczak, M., Brianda M.E., Avalosse, H. \&amp;Roskam, I. (2018b). Consequences of parental burnout: its specific effect on child neglect and violence. Child abuse \&amp;Neglect 80, 134-145. doi: 10.1016/j.chiabu.2018.03.025

18. Griffith, A.K. Parental Burnout and Child Maltreatment During the COVID-19 Pandemic. J

Fam Viol (2020). https://doi.org/10.1007/s10896-020-00172-2

19. Roskam I, Raes M E, Mikolajczak M. Exhausted Parents: Development and Preliminary

Validation of the Parental Burnout Inventory. 2017; 8,163.

20. Van Bakel, H. J. A., Van Engen, M. L., \&amp; Peters, P. (2018). Validity of the parental burnout inventory among dutch employees. Frontiers in Psychology, 9(MAY)

doi:10.3389/fpsyg.2018.00697

21. Ercolani AP, Areni A, Leone L. Statistica per la psicologia. Vol. 2: Statistica inferenziale e analisi dei dati. Il Mulino 2002. 